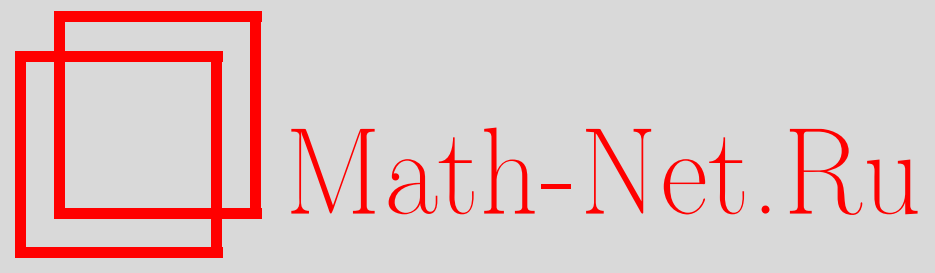

Е. В. Мурашкин, Ю. Н. Радаев, О сильных и слабых разрывах связанного термомеханического поля в термоупругих микрополярных континуумах второго типа, Вестн. Сам. гос. техн. ун-та. Сер. Физ.-мат. науки, 2014, выпуск 4(), 85-97

DOI: https://doi.org/10.14498/vsgtu1331

Использование Общероссийского математического портала MathNet.Ru подразумевает, что вы прочитали и согласны с пользовательским соглашением

http://www.mathnet.ru/rus/agreement

Параметры загрузки:

IP : 54.210 .77 .194

26 апреля 2023 г., $12: 47: 27$

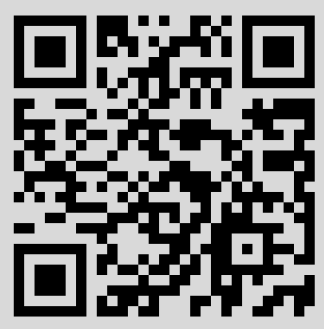


Вестн. Сам. гос. техн. ун-та. Сер. Физ.-мат. науки. 2014. № 4 (37). С. 85-97

ISSN: 2310-7081 (online), 1991-8615 (print)

doi: http://dx.doi.org/10.14498/vsgtu1331

УДК 536.2:539.3

\title{
О СИЛЬНЫХ И СЛАБЫХ РАЗРЫВАХ СВЯЗАННОГО ТЕРМОМЕХАНИЧЕСКОГО ПОЛЯ В ТЕРМОУПРУГИХ МИКРОПОЛЯРНЫХ КОНТИНУУМАХ ВТОРОГО ТИПА*
}

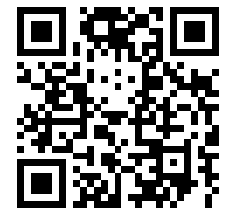

\section{Е. В. Мурашкин, Ю. Н. Радаев}

Институт проблем механики им. А. Ю. Ишлинского РАН, Россия, 119526, Москва, просп. Вернадского, 101, корп. 1.

\begin{abstract}
Аннотация
В работе рассматривается проблема распространения поверхностей сильных и слабых разрывов трансляционных перемещений, микровращений и температурного смещения в микрополярных (MP) термоупругих (TE) континуумах второго типа. Первая часть работы посвящена обсуждению возможного распространения поверхностей сильных разрывов полевых переменных в MPTE-II континуумах. С использованием формализма теории поля получены определяющие соотношения для гиперболического термоупругого микрополярного континуума второго типа. Специальная форма первой вариации функционала действия используется для получения 4-ковариантных условий скачков на волновых поверхностях. Трехмерная форма условий совместности на поверхности сильного разрыва термоупругого поля выводятся из 4-ковариантной формы. Вторая часть работы посвящена проблемам распространения слабых разрывов в MPTE-II континуумах. Для изучения возможных волновых поверхностей слабых разрывов используются геометрические и кинематические условия совместимости Адамара-Томаса. Показано, что поверхности слабых разрывов могут распространяться в отсутствие слабых разрывов температурного смещения.
\end{abstract}

Ключевые слова: микрополярная термоупругость, континуум второго типа, слабый разрыв, сильный разрыв, ударная волна, продольная волна, поперечная волна, условие совместимости, скачок.

doi: http://dx.doi.org/10.14498/vsgtu1331

Вводные замечания. Исследование возможной волновой природы теплопередачи требует привлечения аппарата теории поля [2]. Впервые в полной мере теоретико-полевой подход к построению теории микрополярного континуума был применен в 1909 г. Э. и Ф. Коссера [3]. Впоследствии этот подход получил

(C) 2014 Самарский государственный технический университет.

\section{Образец для цитирования}

М ур ашки н Е. В., Р ад а ев Ю. Н. О сильных и слабых разрывах связанного термомеханического поля в термоупругих микрополярных континуумах второго типа // Becmн. Сам. гос. техн. ун-та. Сер. Физ.-мат. науки, 2014. № 4 (37). С. 85-97. doi: 10.14498/vsgtu1331.

\section{Сведения об авторах}

Евгений Валеръевич Мурашкин (к.ф.-м.н.; murashkin@ipmnet.ru), научный сотрудник, лаб. моделирования в механике деформируемого твердого тела.

Юрий Николаевич Радаев (д.ф.-м.н.; radayev@ipmnet.ru; автор, ведущий переписку), ведущий научный сотрудник, лаб. моделирования в механике деформируемого твердого тела.

*Настоящая статья представляет собой расширенный вариант доклада [1], сделанного авторами на Четвёртой международной конференции «Математическая физика и её приложения» (Россия, Самара, 25 августа - 1 сентября 2014). 
развитие в работе [4]. В монографии [2], терминология и обозначения которой будут использоваться в дальнейшем, развивается вариационный подход к исследованию динамических процессов в связанных микрополярных термоупругих континуумах, основанный на функционале действия. Привлечение подобного формализма при построении математических теорий неизотермического поведения континуумов объясняется невозможностью в рамках классической теории упругости (и пьезоэлектроупругости) объяснить, например, аномальный пьезоэффект в кварце, дисперсию упругих волн, эффект „второго звука“", а также ряд других экспериментально наблюдаемых упругих свойств чистых кристаллов.

В настоящее время быстро развиваются математические модели термоупругого поведения твердых тел (GN-термоупругость), основанные на различных модификациях закона теплопроводности Фурье. При этом ставится цель получения связанных гиперболических уравнений термоупругости, которые гарантировали бы выполнение следующих условий:

1) конечность скорости распространения теплового сигнала;

2) возможность пространственного распространения теплового импульса без затухания;

3) отсутствие искажения волны в смысле получения решений классического даламберовского типа с сохраняющимся профилем.

Одно из таких направлений связано с публикациями $[5,6]$.

1. Функционал действия. Формализм теории поля предполагает использование для математического описания физических полей интегрального функционала действия. Для 4-пространства-времени с элементарным объемом

$$
d^{4} X=d X^{1} d X^{2} d X^{3} d X^{4}
$$

общую форму действия примем в форме

$$
\Im=\int \mathcal{L}\left(X^{\beta}, \varphi^{k}, \partial_{\alpha} \varphi^{k}\right) d^{4} X
$$

где $\varphi^{k}$ - массив физических полей, $\partial_{\alpha}$ - оператор полного дифференцирования по пространственно-временной координате $X^{\alpha}, \alpha=1,2,3,4$.

Принцип наименьшего действия устанавливает, что действительное поле реализуется в 4-пространстве-времени таким образом, что действие (1) оказывается экстремальным, т.е. для всех допустимых вариаций физических полей $\varphi^{k}$ и неварьируемых координат $X^{\alpha}$ имеем $\delta \Im=0$.

Из принципа наименьшего действия получаются классические уравнения Әйлера-Лагранжа

$$
\mathcal{E}_{k}(\mathcal{L})=\frac{\partial \mathcal{L}}{\partial \varphi^{k}}-\partial_{\alpha} \frac{\partial \mathcal{L}}{\partial\left(\partial_{\alpha} \varphi^{k}\right)}=0
$$

В теории поля любой закон сохранения имеет вид $\partial_{\beta} J^{\beta}=0$, где вектор $J^{\beta}$ называется вектором тока. Для него в конечных вариациях можно получить

$$
J^{\beta}=\frac{\partial \mathcal{L}}{\partial\left(\partial_{\beta} \varphi^{k}\right)} \delta^{\nabla} \varphi^{k}+\left(\mathcal{L} \delta_{\alpha}^{\beta}-\left(\partial_{\alpha} \varphi^{k}\right) \frac{\partial \mathcal{L}}{\partial\left(\partial_{\beta} \varphi^{k}\right)}\right) \delta^{\nabla} X^{\alpha}
$$


Вариацию действия, отвечающую конечным вариациям пространственновременных координат и физических полей, можно представить в форме [2]

$$
\delta^{\nabla} \Im=\int\left(\partial_{\beta} J^{\beta}\right) d^{4} X
$$

Будем предполагать, что при переходе через некоторую двустороннюю поверхность $\Sigma^{ \pm}$, распространяющуюся в пространстве с нормальной скоростью $G$, с 4-вектором нормали $\mathcal{N}_{\beta}$ в 4-пространстве-времени термоупругое поле непрерывно, а его первые градиенты по пространственно-временным переменным могут быть разрывными. Квадратными скобками будем обозначать скачки заключенных в них величин при переходе через поверхность $\Sigma$.

2. Условия совместности сильных разрывов. В формуле (2) заменим интеграл по 4-объёму на сумму поверхностных. Закрепляя вариации $\delta \varphi^{k}$ и $\delta X^{\beta}$ на внешней границе поля, можно сохранить лишь два поверхностных интеграла по дважды проходимой поверхности $\Sigma$ :

$$
\delta \Im=\int_{\Sigma^{+}} J^{\beta} \mathcal{N}_{\beta} d \Sigma-\int_{\Sigma^{-}} J^{\beta} \mathcal{N}_{\beta} d \Sigma
$$

Поскольку для действительного поля $\delta \Im=0$, а вариации $\delta \varphi^{k}$ и $\delta X^{\beta}$ непрерывны при переходе через поверхность $\Sigma$, из уравнения (3) получаются следующие 4-ковариантные соотношения совместности сильных разрывов поля:

$$
\mathcal{N}_{\beta}\left[-\frac{\partial \mathcal{L}}{\partial\left(\partial_{\beta} \varphi^{k}\right)}\right]=0, \quad \mathcal{N}_{\beta}\left[\mathcal{L} \delta_{\alpha}^{\beta}-\left(\partial_{\alpha} \varphi^{k}\right) \frac{\partial \mathcal{L}}{\partial\left(\partial_{\beta} \varphi^{k}\right)}\right]=0
$$

Видно, что в уравнениях совместности (4) на поверхности сильного разрыва присутствуют скачки 4-тензора Пиола-Кирхгофа и 4-тензора энергииимпульса поля

$$
S_{4}^{\beta \cdot k}=-\frac{\partial \mathcal{L}}{\partial\left(\partial_{\beta} \varphi^{k}\right)}, \quad T_{\cdot \alpha}^{\beta \cdot}=\mathcal{L} \delta_{\alpha}^{\beta}-\left(\partial_{\alpha} \varphi^{k}\right) \frac{\partial \mathcal{L}}{\partial\left(\partial_{\beta} \varphi^{k}\right)} .
$$

Трехмерные формы условий на поверхности сильного разрыва поля находятся из полученной ковариантной четырехмерной формы:

$$
\begin{aligned}
& -G\left[T_{\cdot 4}^{4 \cdot}\right]+n_{\mu}\left[T_{\cdot 4}^{\mu \cdot}\right]=0, \quad-G\left[T_{\cdot \lambda}^{4 \cdot}\right]+n_{\mu}\left[T_{\cdot \lambda}^{\mu \cdot}\right]=0, \quad \lambda, \mu=1,2,3 ; \\
& -G\left[S_{\cdot}^{4 \cdot k}\right]+n_{\mu}\left[S_{4}^{\mu \cdot k}\right]=0, \quad \mu=1,2,3
\end{aligned}
$$

где $n_{\mu}$ - единичный вектор 3-нормали.

Условия совместности сильных разрывов дополняются известными [7] геометрическими и кинематическими условиями совместности Адамара-Томаса второго и первого порядка, справедливыми для произвольного поля $\varphi^{k}$ и восходящими к Ренкину и Гюгонио [8,9]:

$$
\begin{gathered}
{\left[\partial_{i} \partial_{j} \varphi^{k}\right]=C^{k} n_{i} n_{j}+g^{\alpha \beta} \partial_{\alpha^{\prime}} B^{k}\left(n_{i} \partial_{\beta^{\prime}} x_{j}+n_{j} \partial_{\beta^{\prime}} x_{i}\right)-g^{\alpha \beta} g^{\sigma \tau} B^{k} b_{\alpha \sigma} \partial_{\beta^{\prime}} x_{i} \partial_{\tau^{\prime}} x_{j}} \\
{\left[\partial_{i} \partial_{4} \varphi^{k}\right]=\left(-C^{k} G+\delta_{4} B^{k}\right) n_{i}+g^{\alpha \beta} \partial_{\alpha^{\prime}}\left(B^{k} G\right) \partial_{\beta^{\prime}} x_{i}}
\end{gathered}
$$




$$
\begin{aligned}
{\left[\partial_{4} \partial_{4} \varphi^{k}\right] } & =\left(C^{k} G-\delta_{4} B^{k}\right) G+B^{k} \delta_{4} G, \\
{\left[\partial_{4} \varphi^{k}\right] } & =-B^{k} G, \quad\left[\partial_{i} \varphi^{k}\right]=B^{k} n_{i},
\end{aligned}
$$

где $i, j=1,2,3 ; \alpha, \beta, \tau, \sigma=1,2)$; штрихом обозначены греческие индексы, относящиеся к поверхностным координатам; $C^{k}=\left[\partial_{i} \partial_{j} \varphi^{k}\right] n^{i} n^{j} ; g^{\alpha \beta}$ и $b_{\alpha \beta}-$ компоненты первой и второй фундаментальной формы волновой поверхности соответственно; $\delta_{4}$ - оператор $\delta$-дифференцирования.

3. Действия и условия на разрывах для MPTE-II континуума. Термодинамически корректной теорией, удовлетворяющей перечисленным в первом разделе работы условиям, является теория GNII; в этом случае действие с учетом микроструктуры можно принять в форме [2]

$$
\Im=\int \mathcal{L}\left(X^{\alpha}, x^{j}, d_{\mathfrak{a}}^{j}, \vartheta, \partial_{4} x^{j}, \partial_{4} d_{\mathfrak{a}}^{j}, \partial_{4} \vartheta, \partial_{\beta} x^{j}, \partial_{\beta} d_{\mathfrak{a}}^{j}, \partial_{\beta} \vartheta\right) d X^{1} d X^{2} d X^{3} d X^{4}
$$

Здесь $X^{\alpha}(\alpha=1,2,3)$ - лагранжевы координаты; $x^{j}(j=1,2,3)$ - эйлеровы координаты; $d^{j}(\mathfrak{a}=1,2,3)$ - директоры, связанные с микрообъемом; $\vartheta-$ температурное смещение.

Плотность действия (1) зададим в форме

$$
\begin{aligned}
\mathcal{L}=\frac{1}{2} \rho_{\mathrm{R}} g_{k j} \partial_{4} x^{k} \partial_{4} x^{j}+\frac{1}{2} \rho_{\mathrm{R}} g_{i j} \stackrel{\mathfrak{a} \mathfrak{I}}{\mathfrak{I}} \partial_{4} d_{\mathfrak{a}} d^{i} \partial_{4} d_{\mathfrak{b}}^{j}- & \\
& -\psi\left(X^{\alpha}, x^{j}, d_{\mathfrak{a}}^{j}, \vartheta, \partial_{4} \vartheta, \partial_{\alpha} x^{j}, \partial_{\alpha} d_{\mathfrak{a}}^{j}, \partial_{\alpha} \vartheta\right) .
\end{aligned}
$$

Здесь $\stackrel{\mathfrak{a} \mathfrak{I}}{\mathfrak{I}}$ - тензор микроинерции, $\rho_{\mathrm{R}}$ - референциальная плотность, $g_{i j}$ - метрический тензор пространства, $\psi$ - плотность свободной энергии Гельмгольца.

Уравнения поля в этом случае принимают следующий вид:

$$
\begin{array}{ll}
\partial_{\alpha} S_{\cdot j}^{\alpha \cdot}-\partial_{4} P_{j}=-\frac{\partial \mathcal{L}}{\partial_{x} x^{j}}, & \alpha=1,2,3 ; j=1,2,3 ; \\
\partial_{\alpha} \mathfrak{M}_{\cdot j}^{\alpha}+\stackrel{\mathfrak{A}}{\mathcal{A}}_{j}-\partial_{4}\left(\stackrel{\mathfrak{Q}}{\mathcal{Q}}_{j}\right)=0, & \mathfrak{a}=1,2,3 ; \alpha=1,2,3 ; j=1,2,3 ; \\
\partial_{\alpha} j_{\mathrm{R}}^{\alpha}+\partial_{4} s=\frac{\partial \mathcal{L}}{\partial \vartheta}, & \alpha=1,2,3
\end{array}
$$

и дополняются определяющими уравнениями

$$
\begin{aligned}
& S_{\cdot j}^{\alpha \cdot}=-\frac{\partial \mathcal{L}}{\partial\left(\partial_{\alpha} x^{j}\right)}, \quad \stackrel{\mathfrak{M}}{* j}_{\cdot j}^{\alpha \cdot}=-\frac{\partial \mathcal{L}}{\partial\left(\partial_{\alpha} d_{\mathfrak{a}}^{j}\right)}, \quad \stackrel{\mathfrak{a}}{\mathcal{A}}_{j}=\frac{\partial \mathcal{L}}{\partial d_{\mathfrak{a}}^{j}}, \\
& P_{j}=\frac{\partial \mathcal{L}}{\partial\left(\partial_{4} x^{j}\right)}, \quad \stackrel{\mathfrak{a}}{\mathcal{Q}}_{j}=\frac{\partial \mathcal{L}}{\partial\left(\partial_{4} d^{j}\right)}, \\
& s=\frac{\partial \mathcal{L}}{\partial\left(\partial_{4} \vartheta\right)}, \quad j_{\mathrm{R}}^{\alpha}=\frac{\partial \mathcal{L}^{\mathfrak{a}}}{\partial\left(\partial_{\alpha} \vartheta\right)} .
\end{aligned}
$$

Условия совместности на поверхности сильного разрыва поля в MPTE-II 
континууме, согласно (5), записываются в такой форме:

$$
\begin{aligned}
& -G\left[\mathcal{L}-P_{l} \partial_{4} x^{l}-\stackrel{\mathfrak{a}}{\mathcal{Q}}_{l} \partial_{4} d_{\mathfrak{a}}^{l}-s \partial_{4} \vartheta\right]+n_{\mu}\left[S_{\cdot l}^{\mu \cdot} \partial_{4} x^{l}+\stackrel{\mathfrak{M}}{\mathcal{M}}_{\cdot l}^{\mu \cdot} \partial_{4} d_{\mathfrak{a}}^{l}-j_{\mathrm{R}}^{\mu} \partial_{4} \vartheta\right]=0, \\
& G\left[P_{l} \partial_{\lambda} x^{l}+\stackrel{\mathfrak{Q}}{\mathcal{Q}}_{l} \partial_{\lambda} d_{\mathfrak{a}}^{l}+s \partial_{\lambda} \vartheta\right]+n_{\mu}\left[\mathcal{L} \delta_{\lambda}^{\mu}+S_{\cdot l}^{\mu \cdot} \partial_{\lambda} x^{l}+\stackrel{\mathfrak{M}}{\mathfrak{M}}_{\cdot l}^{\mu \cdot} \partial_{\lambda} d_{\mathfrak{a}}^{l}-j_{\mathrm{R}}^{\mu} \partial_{\lambda} \vartheta\right]=0, \\
& G \rho_{R} g_{k l}\left[\partial_{4} x^{k}\right]=n_{\mu}\left[S_{\cdot l}^{\mu \cdot}\right], \quad G \rho_{R} g_{k l} \stackrel{\mathfrak{a} \mathfrak{I}}{\mathfrak{I}}\left[\partial_{4} d_{\mathfrak{a}}^{k}\right]=n_{\mu}\left[\stackrel{\mathfrak{M}}{\mathcal{M}}_{\cdot l}^{\mu \cdot}\right], \\
& G[s]=n_{\mu}\left[j_{\mathrm{R}}^{\mu}\right], \quad l, \lambda, \mu=1,2,3 \text {. }
\end{aligned}
$$

4. Основные уравнения линейного MPTE-II континуума. Воспользуемся линейной моделью термоупругого микрополярного континуума второго типа (см., например [2,6]). В этом случае векторы перемещений и и микровращений $\phi$ связаны с несимметричным тензором деформации $\boldsymbol{e}$ и тензором изгиба-кручения $\boldsymbol{\Gamma}$ соотношениями

$$
e=\nabla \otimes \mathbf{u}-\epsilon \cdot \phi, \quad \Gamma=\nabla \otimes \phi,
$$

где $\boldsymbol{\epsilon}$ - кососимметричный тензор Леви-Чивита третьего ранга, $\boldsymbol{\nabla}$ - трехмерный оператор Гамильтона (набла Гамильтона).

Несимметричный тензор силовых напряжений $\boldsymbol{\sigma}$ и тензор моментных напряжений $\boldsymbol{m}$ вычисляются согласно определяющему закону термоупругости

$$
\begin{aligned}
& \boldsymbol{\sigma}=(\mu+\eta) \boldsymbol{e}+(\mu-\eta) \boldsymbol{e}^{\mathrm{T}}+(\lambda \operatorname{tr} \boldsymbol{e}-\alpha \theta) \mathbf{I} \\
& \boldsymbol{m}=(\gamma+\varepsilon) \boldsymbol{\Gamma}+(\gamma-\varepsilon) \boldsymbol{\Gamma}^{\mathrm{T}}+(\beta \operatorname{tr} \boldsymbol{\Gamma}-\varsigma \theta) \mathbf{I} .
\end{aligned}
$$

Здесь $\mathbf{I}$ - единичный тензор; $\lambda, \mu, \eta, \gamma, \beta, \varepsilon$ - изотермические определяющие постоянные микрополярной термоупругой среды первого типа; $\alpha$, $\varsigma$ - определяющие постоянные, обеспечивающие связанность уравнений движения и теплопроводности. Постоянные $\alpha, \varsigma$ зависят не только от механических, но и от термических свойств среды.

Уравнения движения микрополярной среды запишем в предположении отсутствия массовых сил и массовых моментов:

$$
\nabla \cdot \sigma=\rho \ddot{\mathbf{u}}, \quad \epsilon \cdot \boldsymbol{\sigma}+\nabla \cdot \boldsymbol{m}=\mathfrak{J} \ddot{\phi},
$$

где $\rho$ - плотность среды, $\mathfrak{J}$ - мера микроинерции среды при вращении, точка над символом обозначает частное дифференцирование по времени при фиксированных пространственных координатах.

Уравнения движения (7) следует дополнить уравнением теплопроводности. Его нетрудно получить, воспользовавшись законом распространения тепла. Теория Грина-Нахди второго типа $[5,6]$ устанавливает пропорциональность вектора скорости потока тепла $\dot{\mathbf{h}}$ и отрицательного градиента температуры $\theta$ :

$$
\dot{\mathbf{h}}=-\Lambda \nabla \theta
$$

где $\Lambda$ - постоянная скорости теплопроводности, $\Lambda>0$. В рамках гиперболической термоупругости преодолевается присущий теории теплопередачи Фурье парадокс о бесконечной скорости распространения тепла.

Если подставить напряжения $\boldsymbol{\sigma}$ и $\boldsymbol{m}$ из формул (6) в уравнения движения (7) и выразить тензоры $\boldsymbol{\Gamma}$ и $\boldsymbol{e}$ через перемещения $\mathbf{u}$ и микровращения $\boldsymbol{\phi}$, то 
получим замкнутую систему связанных уравнений движения и теплопроводности для линейного изотропного центральносимметричного микрополярного термоупругого тела второго типа при условии отсутствия массовых сил, моментов и источников тепла:

$$
\left\{\begin{array}{l}
(\lambda+\mu-\eta) \boldsymbol{\nabla} \boldsymbol{\nabla} \cdot \mathbf{u}+(\mu+\eta) \boldsymbol{\nabla}^{2} \mathbf{u}+2 \eta \boldsymbol{\nabla} \times \boldsymbol{\phi}-\alpha \boldsymbol{\nabla} \theta-\rho \ddot{\mathbf{u}}=\mathbf{0}, \\
(\beta+\gamma-\varepsilon) \boldsymbol{\nabla} \boldsymbol{\nabla} \cdot \boldsymbol{\phi}+(\gamma+\varepsilon) \boldsymbol{\nabla}^{2} \boldsymbol{\phi}-4 \eta \boldsymbol{\phi}+2 \eta \boldsymbol{\nabla} \times \mathbf{u}-\varsigma \boldsymbol{\nabla} \theta-\mathfrak{J} \boldsymbol{\phi}=\mathbf{0}, \\
\boldsymbol{\nabla}^{2} \theta-\frac{\kappa}{\Lambda} \ddot{\theta}-\frac{\alpha \theta_{0}}{\Lambda} \boldsymbol{\nabla} \cdot \ddot{\mathbf{u}}-\frac{\varsigma \theta_{0}}{\Lambda} \boldsymbol{\nabla} \cdot \ddot{\boldsymbol{\phi}}=0 .
\end{array}\right.
$$

Здесь $\nabla^{2}=\boldsymbol{\nabla} \cdot \boldsymbol{\nabla}$ - трехмерный оператор Лапласа, $\theta$ - приращение температуры над отсчетной температурой, $\theta_{0}$ - отсчетная температура, $\Lambda$ - коэффициент теплопроводности (коэффициент термической диффузии), $\kappa$ - теплоемкость (на единицу объема) при постоянной деформации. Отметим, что система дифференциальных уравнений в частных производных (8) содержит частные производные третьего порядка от перемещений и микровращений. Можно получить более симметричную форму уравнений, если ввести вместо $\theta$ температуры температурное смещение $\vartheta$ :

$$
\dot{\vartheta}=\theta
$$

В результате замены переменных получим

$$
\left\{\begin{array}{l}
(\lambda+\mu-\eta) \boldsymbol{\nabla} \boldsymbol{\nabla} \cdot \mathbf{u}+(\mu+\eta) \boldsymbol{\nabla}^{2} \mathbf{u}+2 \eta \boldsymbol{\nabla} \times \boldsymbol{\phi}-\alpha \boldsymbol{\nabla} \dot{\vartheta}-\rho \ddot{\mathbf{u}}=\mathbf{0}, \\
(\beta+\gamma-\varepsilon) \boldsymbol{\nabla} \boldsymbol{\nabla} \cdot \boldsymbol{\phi}+(\gamma+\varepsilon) \boldsymbol{\nabla}^{2} \boldsymbol{\phi}-4 \eta \boldsymbol{\phi}+2 \eta \boldsymbol{\nabla} \times \mathbf{u}-\varsigma \boldsymbol{\nabla} \dot{\vartheta}-\mathfrak{J} \ddot{\boldsymbol{\phi}}=\mathbf{0}, \\
\boldsymbol{\nabla}^{2} \vartheta-\frac{\kappa}{\Lambda} \ddot{\vartheta}-\frac{\alpha \theta_{0}}{\Lambda} \boldsymbol{\nabla} \cdot \dot{\mathbf{u}}-\frac{\varsigma \theta_{0}}{\Lambda} \boldsymbol{\nabla} \cdot \dot{\boldsymbol{\phi}}=0 .
\end{array}\right.
$$

В этом представлении уравнения связанной гиперболической микрополярной термоупругости второго типа содержат частные производные не выше второго порядка.

Как указывалось выше, отличные от нуля определяющие постоянные $\alpha, \varsigma$ обеспечивают связанность уравнений микрополярной термоупругости. Обычно считается, что $\varsigma=0$ [10]. Ради полноты анализа мы сохраним эту определяющую постоянную во всех дальнейших рассуждениях.

Скалярное уравнение в системе (9) называется обобщенным гиперболическим уравнением теплопроводности, сопряженным с уравнениями движения (первое и второе уравнения в системе (9)).

5. Слабые разрывы термомеханических полей в MPTE-II континууме. Исследуем сначала закономерности распространения слабых разрывов перемещений $\mathbf{u}$, микровращений $\phi$ и температурного смещения $\vartheta$ в термоупругом микрополярном континууме второго типа. Отметим, что система дифференциальных уравнений в частных производных (9) содержит частные производные не выше второго порядка. Пусть в трехмерном пространстве с нормальной скоростью $G$ распространяется фронт (волновая поверхность) слабых разрывов перемещений $\mathbf{u}$, микровращений $\phi$ и температурного смещения $\vartheta$. Обозначим через $\mathbf{n}$ единичный вектор нормали к указанной волновой поверхности. Тогда геометрические и кинематические условия совместности второго 
порядка Адамара-Томаса [7] будут иметь вид

$$
\begin{array}{ll}
{[\boldsymbol{\nabla} \otimes \boldsymbol{\nabla} \otimes \mathbf{u}]=\mathbf{n} \otimes \mathbf{n} \otimes \mathbf{A},} & {[\boldsymbol{\nabla} \otimes \boldsymbol{\nabla} \otimes \boldsymbol{\phi}]=\mathbf{n} \otimes \mathbf{n} \otimes \mathbf{S},} \\
{[\boldsymbol{\nabla} \otimes \dot{\mathbf{u}}]=-G \mathbf{n} \otimes \mathbf{A},} & {[\boldsymbol{\nabla} \otimes \dot{\boldsymbol{\phi}}]=-G \mathbf{n} \otimes \mathbf{S},} \\
{[\ddot{\mathbf{u}}]=G^{2} \mathbf{A},} & {[\ddot{\phi}]=G^{2} \mathbf{S},} \\
{[\boldsymbol{\nabla} \otimes \boldsymbol{\nabla} \vartheta]=B \mathbf{n} \otimes \mathbf{n},} & {[\ddot{\vartheta}]=G^{2} B,} \\
{[\boldsymbol{\nabla} \otimes \dot{\vartheta}]=-G B \mathbf{n},} &
\end{array}
$$

где квадратные скобки обозначают скачок при переходе через поверхность слабого разрыва. $B, \mathbf{A}, \mathbf{S}$ - некоторые поля, определенные на этой поверхности, причем равенства $B=0, \mathbf{A}=\mathbf{0}, \mathbf{S}=\mathbf{0}$ не могут выполняться одновременно ни в какой точке поверхности, если рассматриваемая поверхность действительно является поверхностью слабого разрыва. Уравнения (9) вместе с (10) дают следующие соотношения, связывающие скачки частных производных второго порядка от перемещений $\mathbf{u}$, микровращений $\boldsymbol{\phi}$ и температурного смещения $\vartheta$ при переходе через волновую поверхность:

$$
\left\{\begin{array}{l}
\left(\rho G^{2}-(\mu+\eta)\right) \mathbf{A}-(\lambda+\mu-\eta) \mathbf{n}(\mathbf{n} \cdot \mathbf{A})-\alpha G B \mathbf{n}=\mathbf{0} \\
\left(\mathfrak{J} G^{2}-(\gamma+\varepsilon)\right) \mathbf{S}-(\beta+\gamma-\varepsilon) \mathbf{n}(\mathbf{n} \cdot \mathbf{S})-\varsigma G B \mathbf{n}=\mathbf{0} \\
B-\frac{\kappa}{\Lambda} G^{2} B+\frac{\alpha G}{\Lambda} \mathbf{n} \cdot \mathbf{A}+\frac{\varsigma G}{\Lambda} \mathbf{n} \cdot \mathbf{S}=0
\end{array}\right.
$$

Разложим векторы поляризации слабых разрывов на сумму проекций на касательное $\boldsymbol{\tau}$ и нормальное $\mathbf{n}$ направления к волновой поверхности:

$$
\begin{aligned}
& \mathbf{A}=A_{\perp} \boldsymbol{\tau}+A_{\|} \mathbf{n}, \quad \mathbf{S}=S_{\perp} \tau+S_{\|} \mathbf{n} \\
& A_{\perp}=\mathbf{A} \cdot \boldsymbol{\tau}, \quad A_{\|}=\mathbf{A} \cdot \mathbf{n}, \quad S_{\perp}=\mathbf{S} \cdot \boldsymbol{\tau}, \quad S_{\|}=\mathbf{S} \cdot \mathbf{n} .
\end{aligned}
$$

Тогда, учитывая (12), система (11) преобразуется к следующему виду:

$$
\left\{\begin{array}{l}
\left(\rho G^{2}-(\mu+\eta)\right)\left(A_{\perp} \boldsymbol{\tau}+A_{\|} \mathbf{n}\right)-(\lambda+\mu-\eta) A_{\|} \mathbf{n}-\alpha G B \mathbf{n}=\mathbf{0}, \\
\left(\mathfrak{J} G^{2}-(\gamma+\varepsilon)\right)\left(S_{\perp} \boldsymbol{\tau}+S_{\|} \mathbf{n}\right)-(\beta+\gamma-\varepsilon) S_{\|} \mathbf{n}-\varsigma G B \mathbf{n}=\mathbf{0}, \\
B-\frac{\kappa}{\Lambda} G^{2} B+\frac{\alpha G}{\Lambda} A_{\|}+\frac{\varsigma G}{\Lambda} S_{\|}=0 .
\end{array}\right.
$$

Первые два уравнения системы (13) будут справедливы только в случае выполнения следующих условий:

$$
\left\{\begin{array} { l } 
{ ( \rho G ^ { 2 } - ( \mu + \eta ) ) A _ { \perp } = 0 , } \\
{ ( \rho G ^ { 2 } - ( \lambda + 2 \mu ) ) A _ { \| } - \alpha G B = 0 , }
\end{array} \quad \left\{\begin{array}{l}
\left(\mathfrak{J} G^{2}-(\gamma+\varepsilon)\right) S_{\perp}=0, \\
\left(\mathfrak{J} G^{2}-(\gamma+2 \beta)\right) S_{\|}-\varsigma G B=0 .
\end{array}\right.\right.
$$

Обратим внимание на то обстоятельство, что слабые разрывы температурного смещения никак не связаны с касательными проекциями векторов поляризации слабых разрывов $A_{\perp}$ и $S_{\perp}$, что означает возможность распространения поперечных волн в MPTE-II континууме при отсутствии слабых разрывов температурного смещения, как и в аналогичных случаях для MPТЕ-I континуума [10]. При этом скорости распространения таких волновых поверхностей совпадают со скоростями распространения поперечных волн в MPTE-I континууме [10,11]. 
Анализ системы уравнений (15) показывает, что распространение продольных волн в MPTE-II континууме возможно в следующих четырех случаях:

I случай $-A_{\perp} \neq 0, S_{\perp}=0$;

II случай $-A_{\perp}=0, S_{\perp} \neq 0$;

III случай $-A_{\perp} \neq 0, S_{\perp} \neq 0$;

IV случай $-A_{\perp}=0, S_{\perp}=0$.

В первом случае при наличии слабого разрыва трансляционных перемещений $A_{\perp} \neq 0$ выполнение первого уравнения в системе (14) возможно только на поверхности слабого разрыва, распространяющейся с нормальной скоростью

$$
G=c_{\perp}^{\mu}
$$

где

$$
c_{\perp}^{\mu}=\sqrt{\frac{\mu+\eta}{\rho}} .
$$

Заметим, что при равенстве нулю определяющей микрополярной постоянной $\eta$ скорость $G$ в точности совпадет со скоростью чисто упругой поперечной волны $c_{\perp}=\sqrt{\mu / \rho}$.

Во втором случае $S_{\perp} \neq 0$, третье уравнение системы (14) позволяет определить скорость распространения слабого разрыва микровращений

$$
G=c_{\perp}^{\mu \mu}
$$

где

$$
c_{\perp}^{\mu \mu}=\sqrt{\frac{\gamma+\varepsilon}{\mathfrak{J}}} .
$$

В случае одновременного выполнения условий $A_{\perp} \neq 0$ и $S_{\perp} \neq 0$ множители при $A_{\perp}$ и $S_{\perp}$ не могут одновременно обращаться в нуль, если $c_{\perp}^{\mu} \neq c_{\perp}^{\mu \mu}$. Если континуум допускает совпадение скоростей поперечных волн $c_{\perp}^{\bar{\mu}}=c_{\perp}^{\bar{\mu} \mu}$, то скорость распространения волновой поверхности оказывается равной

$$
G=c_{\perp}^{\mu}=c_{\perp}^{\mu \mu}
$$

В четвертом случае распротранение волновой поверхности в MPTE-II континууме возможно только при наличии ненулевых нормальных составляющих векторов поляризации слабых разрывов.

Проанализируем распространение волновых поверхностей слабых разрывов в MPTE-II континууме в отсутствие касательных составляющих векторов поляризации разрывов $\mathbf{A}$ и $\mathbf{S}$. Для анализа воспользуемся системой линейных уравнений

$$
\left\{\begin{array}{l}
\left(\rho G^{2}-(\lambda+2 \mu)\right) A_{\|}-\alpha G B=0 \\
\left(\mathfrak{J} G^{2}-(\gamma+2 \beta)\right) S_{\|}-\varsigma G B=0 \\
\left(1-l^{-2} G^{2}\right) B+\frac{\alpha G}{\Lambda} A_{\|}+\frac{\varsigma G}{\Lambda} S_{\|}=0
\end{array}\right.
$$


где введено обозначение $l^{-2}=\kappa / \Lambda$.

Отметим, что при отсутствии нормальных проекций векторов поляризации слабых разрывов трансляционных перемещений и микровращений распротранение поверхности слабого разрыва температурного смещения невозможно в силу второго и четвертого уравнений системы (14).

Анализ системы уравнений (15) показывает, что распространение продольных волн в MPTE-II континууме возможно в двух следующих случаях:

І случай $-A_{\|} \neq 0, S_{\|} \neq 0, B \neq 0$;

II случай $-A_{\|} \neq 0, S_{\|} \neq 0, B=0$.

Рассмотрим первый случай распространения поверхностей слабых разрывов трансляционных перемещений, микровращений и температурного смещения в MPTE-II континууме. Выразим из первых двух уравнений системы (15) нормальные проекции векторов поляризации разрывов и поставим результат в третье уравнение. Тогда, предполагая, что на волновой поверхности имеется слабый разрыв температурного смещения $(B \neq 0)$, получим уравнение для скорости распространения волновой поверхность вдоль нормали $\mathbf{n}$ :

$$
\left(1-l^{-2} G^{2}\right)+\frac{s^{2} G^{2}}{G^{2}-c_{\|}^{2}}+\frac{s_{\mu}^{2} G^{2}}{G^{2}-c_{\|}^{\mu \mu 2}}=0
$$

Здесь приняты обозначения для скоростей продольных волн:

$$
c_{\|}^{\mu \mu}=\sqrt{\frac{\beta+2 \gamma}{\mathfrak{J}}}, \quad c_{\|}=\sqrt{\frac{\lambda+2 \mu}{\rho}} ;
$$

для постоянных, характеризующих связанность механических и тепловых эффектов,

$$
s^{2}=\frac{\alpha^{2}}{\rho \Lambda}, \quad s_{\mu}^{2}=\frac{\varsigma^{2}}{\rho \Lambda} .
$$

Проведя несложные преобразования уравнения (16), можно получить бикубическое уравнение для определения скорости распространения поверхности слабых разрывов трансляционных перемещений, микровращений и температурного смещения:

$$
\frac{G^{6}}{l^{6}}-\left(s^{2} \frac{c_{\|}^{\mu \mu 2}}{l^{2}}+s_{\mu}^{2} \frac{c_{\|}^{2}}{l^{2}}\right) \frac{G^{4}}{l^{4}}-\left(s^{2}+s_{\mu}^{2}+\frac{c_{\|}^{\mu \mu 2}}{l^{2}}+\frac{c_{\|}^{2}}{l^{2}}\right) \frac{G^{2}}{l^{2}}-\frac{c_{\|}^{\mu \mu 2}}{l^{2}} \frac{c_{\|}^{2}}{l^{2}}=0 .
$$

Введем следующие обозначения для упрощения анализа корней уравнения (17):

$$
a_{4}=s^{2} \frac{c_{\|}^{\mu \mu 2}}{l^{2}}+s_{\mu}^{2} \frac{c_{\|}^{2}}{l^{2}}, \quad a_{2}=s^{2}+s_{\mu}^{2}+\frac{c_{\|}^{\mu \mu 2}}{l^{2}}+\frac{c_{\|}^{2}}{l^{2}}, \quad a_{0}=\frac{c_{\|}^{\mu \mu 2}}{l^{2}} \frac{c_{\|}^{2}}{l^{2}} .
$$

В итоге получим более компактную форму уравнения (16):

$$
\frac{G^{6}}{l^{6}}-a_{4} \frac{G^{4}}{l^{4}}-a_{2} \frac{G^{2}}{l^{2}}-a_{0}=0 .
$$


Заменой

$$
\frac{G^{2}}{l^{2}}=Y+\frac{a_{4}}{3}
$$

можно свести бикубическое уравнение (17) к неполному кубическому уравнению

$$
Y^{3}+p Y+q=0
$$

где коэффициенты уравнения $p$ и $q$ определяются формулами

$$
p=-\left(a_{2}+\frac{a_{4}^{2}}{3}\right), \quad q=-\left(\frac{2}{27} a_{4}^{3}+\frac{1}{9} a_{2} a_{4}+a_{0}\right) .
$$

Решение неполного кубического уравнения (18) можно найти согласно формулам Кардано. Приведем указанное решение в канонической алгебраической форме

$$
Y_{1}=a+b, \quad Y_{2,3}=-\frac{1}{2}(a+b) \pm i \frac{\sqrt{3}}{2}(a-b),
$$

где

$$
a=\sqrt[3]{-\frac{q}{2}+\sqrt{\mathfrak{D}}}, \quad b=\sqrt[3]{-\frac{q}{2}-\sqrt{\mathfrak{D}}}, \quad \mathfrak{D}=\frac{q^{2}}{4}+\frac{p^{3}}{27} .
$$

В случае $\mathfrak{D}>0$ уравнение будет иметь один вещественный корень и два сопряженных комплексных корня, определяемых формулами (19). Вещественное положительное значение нормальной скорости $G$ распространения волновой поверхности будет определятся формулой

$$
\frac{G}{l}=\sqrt{Y_{1}+\frac{a_{4}}{3}}
$$

Во втором случае множители при $A_{\|}$и $S_{\|}$должны одновременно обращаться в нуль, что невозможно, если $c_{\|} \neq c_{\|}^{\mu \mu}$. Ясно, что при этом рассматриваемая поверхность не будет волновой. Если континуум можно охарактеризовать определяющим равенством $c_{\|}=c_{\|}^{\mu \mu}$, то волновая поверхность будет волной, распространяющейся со скоростью

$$
G=c_{\|}=c_{\|}^{\mu \mu}
$$

а нормальные проекции векторов поляризации разрывов, согласно третьему уравнению в системе (15), должны удовлетворять соотношению

$$
\frac{\alpha}{\varsigma}=-\frac{S_{\|}}{A_{\|}}
$$

Отметим, что распространение продольных волн в MPTE-II континуумах возможно только при одновременном выполнении условий $A_{\|} \neq 0$ и $S_{\|} \neq 0$. В отличие от GNII/CTE возможен случай распространения таких возмущений в отсутствие слабого разрыва температурного смещения. 
Благодарности. Работа выполнена при частичной финансовой поддержке РФФИ (проект № 13-01-00139-а «Гиперболические тепловые волны в твердых телах с микроструктурой») и Министерства образования и науки РФ в рамках проектной части государственного задания ФГБОУ ВПО «СамГТУ» (проект № 16.2518.2014/(K)).

\section{ORCID}

Евгений Валерьевич Мурашкин: http://orcid.org/0000-0002-3267-4742

Юрий Николаевич Радаев: http://orcid.org/0000-0002-0866-2151

\section{БИБЛИОГРАФИЧЕСКИЙ СПИСОК}

1. Мурашкин Е. В., Радаев Ю. Н. О сильных и слабых разрывах связанного термомеханического поля в термоупругих микрополярных континуумах второго типа / Четвертая международная конферениия «Математическая физика и ее приложения»: материалы конф.; ред. чл.-корр. РАН И. В. Волович; д.ф.-м.н., проф. В. П. Радченко. Самара: СамГТУ, 2014. С. 261-262.

2. Ковалев В. А., Радаев Ю. Н. Волновые задачи теории поля и термомеханика. Саратов: Изд-во Сарат. ун-та, 2010. 328 с.

3. Cosserat E. et F. Théorie des corps déformables. Paris: Librairie Scientifique A. Hermann et Fils, 1909. 226 pp.

4. Toupin R. A. Theories of elasticity with couple-stress // Arch. Rational Mech. Anal., 1964. vol. 17, no. 2. pp. 85-112. doi : 10.1007/bf00253050.

5. Green A. E., Naghdi P. M. On undamped heat waves in an elastic solid // Journal of Thermal Stresses, 1992. vol. 15, no. 2. pp. 253-264. doi : 10.1080/01495739208946136.

6. Green A. E., Naghdi P. M. Thermoelasticity without energy dissipation // J. Elasticity, 1993. vol. 31, no. 3. pp. 189-208. doi: 10.1007/BF00044969.

7. Thomas T. Plastic flow and fracture in solids. New York: Academic Press, 1961. ix +267 pp.

8. Rankine W. J. M. On the thermodynamic theory of waves of finite longitudinal disturbance // Proceedings of the Royal Society of London, 1869. vol. 18, no. 114-122. pp. 8083. doi: 10.1098/rspl.1869.0025 ; Rankine W. J. M. On the thermodynamic theory of waves of finite longitudinal disturbance// Philosophical Transactions of the Royal Society of London. vol.160. pp. 277-288. doi: 10.1098/rstl.1870.0015 ; Rankine W. J. M. On the thermodynamic theory of waves of finite longitudinal disturbance / Classic Papers in Shock Compression Science / Shock Wave and High Pressure Phenomena, 1998. pp. 133-148. doi : 10.1007/978-1-4612-2218-7_5.

9. Hugoniot P. H. Sur la propagation du mouvement dans les corps et spécialement dans les gaz parfaits (première partie) // Journal de l'École Polytechnique, 1887. vol. 57. pp. 3-97 (In French).

10. Ковалев В. А., Мурашкин Е. В., Радаев Ю. Н. Математическая теория связанных плоских гармонических термоупругих волн в микрополярных континуумах первого типа // Изв. Сарат. ун-та. Нов. сер. Сер. Математика. Механика. Информатика, 2014. T. 14, № 1. C. 77-87.

11. Nowacki W. Theory of Asymmetric Elasticity. Oxford: Pergamon Press, 1986. viii+383 pp.

Поступила в редакцию $03 / \mathrm{X} / 2014$;

в окончательном варианте - 10/XI/2014;

принята в печать $-27 / \mathrm{XI} / 2014$. 
Vestn. Samar. Gos. Techn. Un-ta. Ser. Fiz.-mat. nauki

[J. Samara State Tech. Univ., Ser. Phys. \& Math. Sci.] 2014. Issue 4 (37). Pp. 85-97

ISSN: 2310-7081 (online), 1991-8615 (print)

doi: http://dx.doi.org/10.14498/vsgtu1331

MSC: 74A15, 74F05, 74B99, 80A20

\title{
ON STRONG AND WEAK DISCONTINUITIES OF THE COUPLED THERMOMECHANICAL FIELD IN MICROPOLAR THERMOELASTIC TYPE-II CONTINUA*
}

\author{
Murashkin E. V., Radayev Yu. N. \\ A. Ishlinsky Institite for Problems in Mechanics, Russian Academy of Sciences, \\ 101, pr. Vernadskogo, Moscow, 119526, Russian Federation.
}

\begin{abstract}
The present study is devoted to problem of propagating surfaces of weak and strong discontinuities of translational displacements, microrotations and temperature in micropolar (MP) thermoelastic (TE) type-II continua. First part of the paper is concerned to discussions of the propagating surfaces of strong discontinuities of field variables in type-II MPTE continua. Constitutive relations for hyperbolic thermoelastic type-II micropolar continuum is derived by the field theory. The special form of the first variation of the action integral is used in order to obtain 4-covariant jump conditions on wave surfaces. Three-dimensional form of the jump conditions on the surface of a strong discontinuity of thermoelastic field are derived from 4-covariant form. Problems of propagation of weak discontinuities in type-II MPTE continua are discussed too. Geometrical and kinematical compatibility conditions due to Hadamard and Thomas are used to study possible wave surfaces of weak discontinuities. It is shown that the surfaces of weak discontinuities can propagate exist without weak discontinuities of the temperature field.
\end{abstract}

Keywords: micropolar thermoelasticity, type-II continuum, weak discontinuity, strong discontinuity, shock wave, longitudinal wave, transverse wave, compatibility condition, jump.

doi: http://dx.doi.org/10.14498/vsgtu1331

Acknowledgments. This work has been partially supported by the Russian Foundation for Basic Research (project no. 13-01-00139-a "Hyperbolic Thermal Waves in Solid Bodies with Microstructure") and by the Russian Ministry of Education and Science within the design basis portion of the state task to Samara State Technical University (project no. 16.2518.2014/(K)).

(C) 2014 Samara State Technical University.

\section{How to cite Reference}

Murashkin E. V., Radayev Yu. N. On strong and weak discontinuities of the coupled thermomechanical field in micropolar thermoelastic type-II continua, Vestn. Samar. Gos. Tekhn. Univ., Ser. Fiz.-Mat. Nauki [J. Samara State Tech. Univ., Ser. Phys. \& Math. Sci.], 2014, no. 4(37), pp. 85-97. doi: 10.14498/vsgtu1331. (In Russian)

Authors Details

Evgenii V. Murashkin (Cand. Phys. \& Math. Sci.; murashkin@ipmnet.ru), Researcher, Lab. of Modeling in Solid Michanics.

Yuri N. Radayev, Dr. Phys. \& Math. Sci.; radayev@ipmnet.ru; Corresponding Author, Leader Researcher, Lab. of Modeling in Solid Michanics.

${ }^{*}$ This paper is an extended version of the paper [1], presented at the Mathematical Physics and Its Applications 2014 Conference. 


\section{ORCID}

Evgenii V. Murashkin: http://orcid.org/0000-0002-3267-4742

Yuri N. Radayev: http://orcid.org/0000-0002-0866-2151

\section{REFERENCES}

1. Murashkin E. V., Radayev Yu. N. On Strong and Weak Discontinuities of the Coupled Thermomechanical Field in Micropolar Thermoelastic Type-II Continua, The 4nd International Conference "Mathematical Physics and its Applications", Book of Abstracts and Conference Materials; eds. I. V. Volovich; V. P. Radchenko. Samara, Samara State Technical Univ., 2014, pp. 261-262 (In Russian).

2. Kovalev V. A., Radayev Yu. N. Volnovye zadachi teorii polia i termomekhanika [Wave problems of the field theory and thermomechanic]. Saratov, Saratov Univ. Publ., 2010, 328 pp. (In Russian)

3. Cosserat E. et F. Théorie des corps déformables. Paris, Librairie Scientifique A. Hermann et Fils, 1909, 226 pp.

4. Toupin R. A. Theories of elasticity with couple-stress, Arch. Rational Mech. Anal., 1964, vol. 17, no. 2, pp. 85-112. doi: 10.1007/bf00253050.

5. Green A. E., Naghdi P. M. On undamped heat waves in an elastic solid, Journal of Thermal Stresses, 1992, vol.15, no. 2, pp. 253-264. doi : 10.1080/01495739208946136.

6. Green A. E., Naghdi P. M. Thermoelasticity without energy dissipation, J. Elasticity, 1993, vol. 31, no. 3, pp. 189-208. doi: 10.1007/BF00044969.

7. Thomas T. Plastic flow and fracture in solids. New York, Academic Press, 1961, ix +267 pp.

8. Rankine W. J. M. On the thermodynamic theory of waves of finite longitudinal disturbance, Proceedings of the Royal Society of London, 1869, vol.18, no. 114-122, pp. 80-83. doi: 10 . 1098/rspl.1869.0025 ; Rankine W. J. M. On the thermodynamic theory of waves of finite longitudinal disturbance, Philosophical Transactions of the Royal Society of London, vol.160, pp. 277-288. doi:10.1098/rstl.1870.0015 ; Rankine W. J. M. On the thermodynamic theory of waves of finite longitudinal disturbance, Classic Papers in Shock Compression Science, Shock Wave and High Pressure Phenomena, 1998, pp. 133148. doi : 10.1007/978-1-4612-2218-7_5.

9. Hugoniot P. H. Sur la propagation du mouvement dans les corps et spécialement dans les gaz parfaits (première partie), Journal de l'École Polytechnique, 1887, vol. 57, pp. 3-97 (In French).

10. Kovalev V. A., Murashkin E. V., Radayev Yu. N. A mathematical theory of plane harmonic coupled thermoelastic waves in type-I micropolar continua, Izv. Saratov. Univ. (N.S.), Ser. Math. Mech. Inform., 2014, vol. 14, no. 1, pp. 77-87 (In Russian).

11. Nowacki W. Theory of Asymmetric Elasticity. Oxford, Pergamon Press, 1986, viii+383 pp.

Received 03/X/2014;

received in revised form $10 / \mathrm{XI} / 2014$;

accepted 27/XI/2014. 